Fermilab-PUB-97/389

June 25, 2018

\title{
Neutrino Beams from Muon Storage Rings: Characteristics and Physics Potential
}

\author{
S. Geer \\ Fermi National Accelerator Laboratory \\ P.O. Box 500, Batavia, Illinois 60510
}

\begin{abstract}
High-intensity high-energy neutrino beams could be produced by exploiting a very intense future muon source, and allowing the muons to decay in a storage ring containing a long straight section. Taking the parameters of muon source designs that are currently under study, the characteristics of the neutrino beams that could be produced are discussed and some examples of their physics potential given. It is shown that the neutrino and antineutrino beam intensities may be sufficient to produce hundreds of charged current interactions per year in a detector on the far side of the Earth.
\end{abstract}

PACS numbers: 14.60.Pq, 13.15.+g, 13.35.Bv, 07.77Ka

Submitted to Physical Review D. 


\section{Introduction}

High energy neutrino beams have played an important role in the development of particle physics. Experiments using neutrino and antineutrino beams produced from charged meson decays have, for example, demonstrated that muon neutrinos are different from electron neutrinos, discovered neutral currents, provided measurements of the structure of the nucleon via deep inelastic scattering, made precision tests of the Standard Model of electroweak interactions via measurements of charged and neutral current interactions, and provided increasingly sensitive searches for neutrino oscillations in short- and long-baseline experiments.

Recent results from atmospheric neutrino, solar neutrino, and short-baseline accelerator neutrino experiments indicate that neutrino oscillations may occur at rates which are within reach of the next generation of accelerator based experiments. It therefore seems certain that experiments utilizing neutrino and antineutrino beams will continue to make important contributions to particle physics, initially by clarifying whether the existing results are indeed indications that neutrinos oscillate, and perhaps eventually by making precise measurements of the oscillations. In addition, there continues to be interest in using neutrino beams to further probe the structure of the nucleon.

The long-term future of the experimental program at neutrino beam facilities will require the continued improvement of the intensity and/or quality of the beams. The present generation of high-energy neutrino beams are made by allowing charged pions and kaons to decay in-flight in a long decay channel. This paper describes how a new type of neutrino beam could be made by exploiting a very high intensity muon source of the type that is currently being designed as part of the effort to develop the technology for a high-luminosity muon collider [1, 2]. The muons would be stored and allowed to decay in a ring containing a long straight section that points in the desired direction. The advantages of producing a neutrino beam using muon decays are discussed in Section 2. The method of producing a neutrino beam from decaying muons is described in Section 3. The resulting calculated fluxes, which are sufficient to provide significant interaction rates in a detector on the far side of the Earth, are described in Section 4 for short-, long-, and very-long baseline neutrino experiments. We note that geophysical applications of a facility that can shoot neutrino beams through the Earth have been discussed in Ref. [3]. Some examples of how the neutrino beams from a high intensity muon storage ring might be used to search for or measure neutrino oscillations are given in Section 5, and conclusions are summarized in Section 6 .

\section{Meson Decays versus Muon Decays}

Presently operating high-energy neutrino beams are made by allowing charged pions and kaons to decay in a long decay channel. Shielding downstream of the decay channel removes the undecayed mesons, but transmits the weakly 
interacting neutrinos to form a "pure" neutrino beam. If positively charged pions and kaons have been selected for the decay channel, the resulting beam downstream of the shielding will contain mostly muon neutrinos produced in the two-body decays $\pi^{+} \rightarrow \mu^{+} \nu_{\mu}$ and $K^{+} \rightarrow \mu^{+} \nu_{\mu}$. The neutrino beam will also contain a small component of electron neutrinos produced in the three-body decays $K^{+} \rightarrow e^{+} \pi^{0} \nu_{e}$. In addition, if the primary proton energy is sufficiently high, the beam will contain a small $\nu_{\tau}$ component coming predominantly from prompt tauonic decays of $D_{s}$ mesons. Antineutrino beams can be made by using a negatively charged meson beam.

Thus, present neutrino (antineutrino) beams consist mostly of muon neutrinos (antineutrinos) with a small $\mathrm{O}(1 \%)$ mixture of electron neutrinos (antineutrinos), and if the beam energy is high enough, a small component of tau neutrinos (antineutrinos). In general, this beam composition is not ideal for neutrino experiments. In particular:

(i) The finite precisions with which the $\nu_{e}$ and $\nu_{\mu}$ fluxes can be determined are important sources of systematic uncertainty for many neutrino experiments.

(ii) The small $\nu_{e}$ contamination in the otherwise pure $\nu_{\mu}$ beam is a nuisance for experiments searching for $\nu_{e}-\nu_{\mu}$ oscillations.

(iii) The smallness of the $\nu_{e}$ flux makes $\nu_{e}-\nu_{\tau}$ oscillation searches difficult, and

(iv) The small $\nu_{\tau}$ contamination in the beam will eventually become a nuisance for experiments searching for $\nu_{\mu}-\nu_{\tau}$ oscillations.

These difficulties can be overcome if the neutrino beam is produced by allowing muons to decay in the straight section of a storage ring. This would produce a beam with a precisely known mixture of neutrino types; namely $50 \%$ muon neutrinos and $50 \%$ electron antineutrinos if a $\mu^{-}$beam is stored, and $50 \%$ muon antineutrinos and $50 \%$ electron neutrinos if a $\mu^{+}$beam is used.

In the muon rest-frame the distribution of muon antineutrinos (neutrinos) from the decay $\mu^{ \pm} \rightarrow e^{ \pm}+\nu_{e}\left(\bar{\nu}_{e}\right)+\bar{\nu}_{\mu}\left(\nu_{\mu}\right)$ is given by the expression 4 :

$$
\frac{d^{2} N_{\nu_{\mu}}}{d x d \Omega}=\frac{2 x^{2}}{4 \pi}[(3-2 x) \mp(1-2 x) \cos \theta]
$$

where $x \equiv 2 E_{\nu} / m_{\mu}, \theta$ is the angle between the neutrino momentum vector and the muon spin direction, and $m_{\mu}$ is the muon rest mass. The corresponding expression describing the distribution of electron neutrinos (antineutrinos) is:

$$
\frac{d^{2} N_{\nu_{e}}}{d x d \Omega}=\frac{12 x^{2}}{4 \pi}[(1-x) \mp(1-x) \cos \theta] .
$$

Thus, the neutrino and antineutrino energy- and angular- distributions depend upon the parent muon energy, the decay angle, and the direction of the muon spin vector. For an ensemble of muons we must average over the polarization of the initial state muons, and the distributions become:

$$
\frac{d^{2} N_{\nu_{\mu}}}{d x d \Omega} \propto \frac{2 x^{2}}{4 \pi}\left[(3-2 x) \mp(1-2 x) P_{\mu} \cos \theta\right],
$$


and

$$
\frac{d^{2} N_{\nu_{e}}}{d x d \Omega} \propto \frac{12 x^{2}}{4 \pi}\left[(1-x) \mp(1-x) P_{\mu} \cos \theta\right],
$$

where $P_{\mu}$ is the average muon polarization along the chosen quantization axis, which in this case is the beam direction.

The advantages of producing a neutrino beam using muon decays rather than meson decays are, therefore:

(a) The absolute neutrino fluxes can be easily and precisely calculated, provided the stored muon current, momentum, and polarization are carefully measured.

(b) Only one type of neutrino and one type of antineutrino are present in the beam, and these types can be chosen by selecting the charge of the stored muons. Thus precise $\nu_{e}, \nu_{\mu}, \bar{\nu}_{e}$, and $\bar{\nu}_{\mu}$ measurements can be made.

In addition, the muons can be polarized and the time dependence of the precessing muon spin vectors monitored, enabling measurements to be made as the differential spectra of the neutrinos and antineutrinos in the beam vary in a precisely known way.

\section{$3 \quad$ Using a Muon Storage Ring}

The muon lifetime is about 100 times longer than the corresponding charged pion lifetime. For example, $20 \mathrm{GeV} / \mathrm{c}$ muons have a decay length $\gamma \mathrm{c} \tau=126 \mathrm{~km}$. Thus, a linear decay channel of the type used to produce conventional neutrino beams would in practice be too short to use efficiently as a muon decay channel. This problem can be overcome by using a muon storage ring with a straight section pointing towards the desired experimental area. For simplicity we will consider a storage ring that consists of two parallel straight sections connected together by two arcs. If the straight sections are long compared to the arc lengths, the circulating muons spend approaching $50 \%$ of their time traveling in the straight section pointing towards the experiment. In practice there is an advantage in keeping the size of the storage ring small. Therefore, in the following we will assume that the straight sections are equal in length to the arcs, and that $25 \%$ of the injected muons decay as they circulate in the ring whilst they are in the straight section pointing at the experiment.

To understand how a muon storage ring designed for a given muon momentum might be used as a neutrino source, and calculate the parameters of the resulting neutrino beams, we must understand some of the basic parameters of the muon source and storage ring, namely the divergence of the beam in the straight sections, the size of the ring, and the number of muons available from the source.

If the ring lattice is properly designed, a beam divergence $\theta_{b} \leq O\left(10^{-4}\right)$ should be achievable [5] in the straight section. Thus, if the circulating muons have momentum $p / m_{\mu} \ll 10^{4}$ (corresponding to $p \ll 1000 \mathrm{GeV} / \mathrm{c}$ ) the angular 
divergence of the neutrino beam produced from decays in the straight sections will be dominated by the decay kinematics.

In the scheme we are considering, the size of the storage ring is determined by the length of the arcs. If the ring is designed to store muons of momentum $\mathrm{p}(\mathrm{GeV} / \mathrm{c})$, the length of each arc in meters is given by:

$$
L=\frac{\pi p}{0.3 f B}
$$

where $B$ is the field of the arc dipole magnets (Tesla) and the "packing fraction" $f$ is the fraction of the arc lengths occupied by the dipoles. As an example, choosing the reasonable values $f=0.7$ and $B=8 \mathrm{~T}$ to store $20 \mathrm{GeV} / \mathrm{c}$ muons we obtain an estimate of $37 \mathrm{~m}$ for the arc lengths and $150 \mathrm{~m}$ for the ring circumference (two arcs and two straight sections). Thus, for muons in this momentum range, it would appear that the required storage ring would be sufficiently compact to contemplate building the ring in a plane tilted at a large angle with respect to the horizon. This would enable the neutrino beams to be directed through the Earth for a very long baseline neutrino oscillation experiment. The estimate we are using for the arc lengths has been confirmed by a study [6] of storage ring lattices for rings designed to store muons with momenta from $10 \mathrm{GeV} / \mathrm{c}$ up to $250 \mathrm{GeV} / \mathrm{c}$.

The direction of the muon beam must be carefully monitored within the straight section to avoid significant systematic uncertainties on the calculated neutrino fluxes at the experiment. This can be done by placing beam position monitors (for example wire chambers) within the muon beam at either ends of the straight section. The angular precision that would be achieved $\left(\sigma_{\theta}\right)$ would depend upon the spatial resolution of the beam position monitors $\left(\sigma_{x}\right)$, and the distance between the monitors (L). Using Eq. 5 to relate L to the stored muon momentum we obtain:

$$
\sigma_{\theta} \sim \frac{0.3 f B}{\pi p} \sqrt{2} \sigma_{x}
$$

where $\sigma_{x}$ is in meters. Hence, for $\mathrm{B}=8 \mathrm{~T}, \mathrm{f}=0.7$, and choosing $\sigma_{x}=500 \mu \mathrm{m}$ we obtain for $\mathrm{p}=20 \mathrm{GeV} / \mathrm{c}$ the estimate $\sigma_{\theta}=19 \mu \mathrm{r}$, which is small compared to the anticipated divergence of the muon beam within the straight section.

The rate at which muons are stored in the ring, and therefore the average neutrino beam intensity, will be determined by the performance of the muon source. In the following we will assume a muon source of the type being developed as part of an ongoing effort to determine the feasibility of building a high-luminosity muon collider [1, 2]. The muon source consists of a proton accelerator, charged pion production target and collection system, pion decay channel, and muon cooling channel. Details of the proton accelerator design are given in Ref. [7], and descriptions of the other components are given in Ref. [1]. For completeness, a brief overview of the muon source and its assumed performance is given in the following paragraph.

In the muon collider front-end design that we are taking as an example, the muon source receives protons from an accelerator complex that accelerates bunches containing $5 \times 10^{13}$ particles to energies of $16 \mathrm{GeV}$. The protons interact 
in a target to produce approximately $3 \times 10^{13}$ charged pions of each sign per proton bunch. These pions are produced with only a very limited component of momentum transverse to the incident proton direction. The charged pions can therefore be confined within a beam channel using, for example, a 20 Tesla co-axial solenoid with an inner radius of $7.5 \mathrm{~cm}$. To collect as many pions as possible within a useful energy interval, it is proposed to use rf cavities to accelerate the lower energy particles and decelerate the higher energy particles. Muons are produced by allowing the pions to decay. At the end of a $20 \mathrm{~m}$ long decay channel, consisting of a 7 Tesla solenoid with a radius of $25 \mathrm{~cm}$, on average 0.2 muons of each charge would be produced for each proton incident on the pion production target. With two proton bunches every accelerator cycle, the first used to make and collect positive muons and the second to make and collect negative muons, there would be about $1 \times 10^{13}$ muons of each charge available at the end of the decay channel per accelerator cycle. If the proton accelerator is cycling at $15 \mathrm{~Hz}$, in an operational year $\left(10^{7}\right.$ secs $)$ about $1.5 \times 10^{21}$ positive and negative muons would have been produced in the decay channel and collected. The muons exiting the decay channel populate a very diffuse phase space. The next step is to "cool" the muon bunch, i.e. to turn the diffuse muon cloud into a very bright bunch with small dimensions in six-dimensional phase-space, suitable for accelerating and injecting into a muon storage ring. The proposed method of cooling the muons is to use ionization cooling [8]. At the end of the ionization cooling channel each muon bunch is expected to contain about $5 \times 10^{12}$ muons with a momentum of order $100 \mathrm{MeV} / \mathrm{c}$. We will assume that the losses in accelerating the muons to modest energies (up to a few $\times 10 \mathrm{GeV}$ or less) are small, and therefore that $7.5 \times 10^{20}$ muons of the desired charge are injected into the storage ring each operational year. In the scheme we are considering, $25 \%$ of the muons will decay in the straight section pointing at the experimental area, and the resulting neutrino beam will contain $2 \times 10^{20}$ neutrinos per year and $2 \times 10^{20}$ antineutrinos per year, with energyand angular-distributions described by Eqs. (1) and (2).

\section{Fluxes and Interaction Rates}

In the following we will consider three scenarios, namely (i) a very long baseline experiment in which the neutrino beam passes through the Earth, (ii) a long baseline experiment in which the neutrino beam dips down a few degrees with respect to the horizon, and (iii) a "near" experiment which is $1 \mathrm{~km}$ from the neutrino source.

\subsection{Very Long Baseline Experiment}

Consider a geometry in which the plane of the storage ring dips at an angle of $51^{\circ}$ to the horizon, and the resulting neutrino beam exits the Earth at the "far site" after traversing $9900 \mathrm{~km}$. This geometry would correspond to a storage 
ring sited at the Fermi National Accelerator Laboratory in the United States with the far site at the Gran Sasso underground Laboratory in Italy.

The calculated neutrino and antineutrino fluxes at the far site are shown in Fig. 1 as a function of the energy and average polarization of the muons decaying in the straight section of the storage ring. The fluxes have been averaged over a $1 \mathrm{~km}$ radius "spot" at the far site. The electron neutrino and antineutrino fluxes are very sensitive to the muon spin direction. The reason for this can be understood by examining Eq. 2 which shows that for $\mu^{+}\left(\mu^{-}\right)$ decays the $\nu_{e}\left(\bar{\nu}_{e}\right)$ flux $\rightarrow 0$ for all neutrino energies as $\cos \theta \rightarrow+1(-1)$.

As an example we will consider in more detail neutrino beams from unpolarized positive muons stored with momenta $\mathrm{p}=20 \mathrm{GeV} / \mathrm{c}(50 \mathrm{GeV} / \mathrm{c})$ which, in the absence of neutrino oscillations, produce at the far site $2.2 \times 10^{10}\left(1.4 \times 10^{11}\right)$ $\bar{\nu}_{\mu} \mathrm{m}^{-2}$ year $^{-1}$ and $2.2 \times 10^{10}\left(1.4 \times 10^{11}\right) \quad \nu_{e} \mathrm{~m}^{-2}$ year $^{-1}$. These results assume that the neutrino beam is pointing exactly in the direction of the far site. The differential distributions $d N_{\nu} / d E_{\nu}$ are shown in Fig. 2 as a function of the angle $\Delta \theta$ between the neutrino beam direction and the direction of the far site. As $\Delta \theta$ increases, both the maximum neutrino energy and the neutrino flux decrease. However if, as expected, the beam direction can be monitored with a precision $\sigma_{\theta} \ll 1 \mathrm{mr}$, the systematic uncertainty on the predicted neutrino and antineutrino fluxes and differential distributions at the far site should be modest.

The charged current neutrino and antineutrino rates in a detector at the far site can be calculated using the approximate expressions [10] for the crosssections:

$$
\sigma_{\nu N} \sim 0.67 \times 10^{-38} \mathrm{~cm}^{2} \times E_{\nu}(\mathrm{GeV})
$$

and

$$
\sigma_{\bar{\nu} N} \sim 0.34 \times 10^{-38} \mathrm{~cm}^{2} \times E_{\bar{\nu}}(\mathrm{GeV}) .
$$

The predicted charged current interaction rates are shown in Fig. 3 as a function of the energy and average polarization of the decaying muons, and the associ-

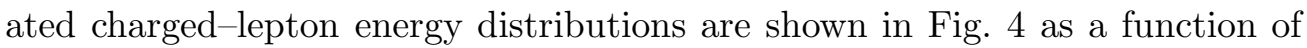
the angle $\Delta \theta$ between the beam direction and the direction of the far site. In the absence of neutrino oscillations, the number of charged current interactions in a $10 \mathrm{kT}$ far site detector $(\Delta \theta=0)$ when unpolarized $20 \mathrm{GeV} / \mathrm{c}(50 \mathrm{GeV} / \mathrm{c})$ positive muons are stored in the ring are $610\left(1.0 \times 10^{4}\right) \bar{\nu}_{\mu}$ interactions per year and $1 \times 10^{3}\left(1.6 \times 10^{4}\right) \nu_{e}$ interactions per year. We conclude that, for these particular examples, interactions from neutrinos and antineutrinos should be readily detectable at the far site. Note that the predicted charged current interaction rates and the shapes of the associated lepton energy spectra are both sensitive to $\Delta \theta$. This could be exploited by locating one or more satellite detectors at angular distances $\Delta \theta=\mathrm{O}(1 \mathrm{mr})$ from the main far site detector.

\subsection{Long Baseline Experiment}

Consider a long baseline geometry in which the plane of the muon storage rings tilts at just a few degrees to the horizon. To be explicit we will consider a far site 
that is $732 \mathrm{~km}$ from the neutrino source. This geometry would correspond [11] to a storage ring sited at the Fermi National Accelerator Laboratory and a far site at the Soudan underground Laboratory in Minnesota.

The neutrino fluxes and charged current interaction rates at the far site can be obtained by scaling the results presented in Figs. 1 and 3 by a factor of 183. Thus, in the absence of neutrino oscillations, unpolarized positive muons stored with momenta $\mathrm{p}=20 \mathrm{GeV} / \mathrm{c}(50 \mathrm{GeV} / \mathrm{c})$ will produce at the far site $4 \times 10^{12}\left(2.6 \times 10^{13}\right) \bar{\nu}_{\mu}$ and $\nu_{e} \mathrm{~m}^{-2}$ year $^{-1}$. In a $10 \mathrm{kT}$ detector, these fluxes would result in $1.1 \times 10^{5}\left(1.8 \times 10^{6}\right) \bar{\nu}_{\mu}$ charged current interactions per year and $1.8 \times 10^{5}\left(2.9 \times 10^{6}\right) \nu_{e}$ charged current interactions per year.

Given these large interaction rates it is worthwhile considering using a lower energy muon storage ring. Predicted fluxes and spectra corresponding to using $10 \mathrm{GeV} / \mathrm{c}$ stored muons are shown in Fig. 5. The calculated neutrino and antineutrino fluxes are both $\sim 1 \times 10^{12} \mathrm{~m}^{-2}$ year $^{-1}$, and the corresponding charged current interaction yields are $3.1 \times 10^{3} \mu^{-} \mathrm{kT}^{-1}$ year ${ }^{-1}$ and $1.4 \times$ $10^{3} e^{+} \mathrm{kT}^{-1}$ year $^{-1}$ when negative muons are stored in the ring, and $1.6 \times$ $10^{3} \mu^{+} \mathrm{kT}^{-1}$ year $^{-1}$ and $2.9 \times 10^{3} e^{-} \mathrm{kT}^{-1}$ year $^{-1}$ when positive muons are stored in the ring. The mean energies of the charged leptons and antileptons produced in these charged current interactions are respectively $\sim 3.5 \mathrm{GeV}$ and $\sim 2 \mathrm{GeV}$. Thus, neutrino and antineutrino charged current interactions should be readily detectable at the far site when the decaying muons in the storage ring have momenta as low as $10 \mathrm{GeV} / \mathrm{c}$.

\subsection{Near Experiment}

Next consider a short baseline geometry in which the detector is $1 \mathrm{~km}$ from the neutrino source. This geometry is of interest to short baseline neutrino oscillation experiments if the neutrino energies are much lower than those considered so far, and of interest to deep inelastic scattering experiments if the neutrino energies are much higher than considered so far.

Consider first a $1.5 \mathrm{GeV} / \mathrm{c}$ muon storage ring. Averaging the fluxes at the detector over a "spot" with a radius of $5 \mathrm{~m}$, the predicted neutrino and antineutrino fluxes resulting from unpolarized muon decays are both $1.2 \times 10^{16} \mathrm{~m}^{-2}$ per year. The corresponding charged current interaction rates in a $1 \mathrm{kT}$ detector yield $2.9 \times 10^{6} \mu^{+}$per year and $5.1 \times 10^{6} e^{-}$per year if positive muons are stored in the ring, and $6.0 \times 10^{6} \mu^{-}$per year and $2.6 \times 10^{6} e^{+}$per year if negative muons are stored in the ring. The calculated neutrino and antineutrino differential spectra are shown in Fig. 6 together with the charged lepton distributions from charged current interactions. A $1 \mathrm{kT}$ detector would record millions of charged current interactions per year with mean charged-lepton energies of $\sim 0.6 \mathrm{GeV}$ and mean charged-antilepton energies of $\sim 0.3 \mathrm{GeV}$.

Finally we consider a higher energy storage ring suitable for deep inelastic scattering experiments. This might be the last recirculating linear accelerator ring in a future muon collider accelerator complex. We will assume the muons are unpolarized and choose $250 \mathrm{GeV} / \mathrm{c}$ for the stored muon momentum. After 
$1 \mathrm{~km}$ the neutrino beam "spot" has a radius of less than $1 \mathrm{~m}$. Hence nearly all of the $\mathrm{O}\left(10^{20}\right)$ neutrinos and antineutrinos per year will pass through a reasonably compact detector. This, together with the very large neutrino flux, would make possible a small detector incorporating detailed tracking and particle identification. If, for example, the fiducial mass is $10 \mathrm{~kg}$, then the estimated charged current interaction rate is $8 \times 10^{5}$ neutrino interactions per year and $5 \times 10^{5}$ antineutrino interactions per year.

\section{$5 \quad$ Examples}

To illustrate the physics potential of the muon storage ring neutrino sources discussed in the previous section, consider the sensitivity of an experiment searching for $\nu_{e}-\nu_{\mu}$ or $\nu_{e}-\nu_{\tau}$ oscillations performed by searching for charged current interactions producing "wrong-sign" muons. For example, if positive muons are stored at the neutrino source, unoscillated muon-antineutrino and electron-neutrino charged current interactions will produce $\mu^{+}$and $e^{-}$respectively. However, if the $\nu_{e}$ transforms itself into a $\nu_{\mu}$ during its passage to the detector the charged current interaction will produce a $\mu^{-}$. Similarly, if the $\nu_{e}$ transforms itself into a $\nu_{\tau}$ and the neutrino energy is sufficiently large, the charged current interaction will produce a $\tau^{-}$which, with a branching ratio of $17 \%$ [9], will decay to produce a $\mu^{-}$.

Within the framework of two-flavor vacuum oscillations, the probability that, whilst traversing a distance $\mathrm{L}$, a neutrino of type 1 (mass $m_{1}$ ) oscillates into a neutrino of type $2\left(\operatorname{mass} m_{2}\right)$ is given by [4]:

$$
P\left(\nu_{1} \rightarrow \nu_{2}\right)=\sin ^{2}(2 \theta) \sin ^{2}\left(1.27 \Delta m^{2} L / E\right),
$$

where $\theta$ is the mixing angle, and $\Delta m^{2} \equiv m_{2}^{2}-m_{1}^{2}$ is measured in $e V^{2} / c^{4}, \mathrm{~L}$ in $\mathrm{km}$, and the neutrino energy $\mathrm{E}$ is in $\mathrm{GeV}$. Hence, in the absence of backgrounds or systematic uncertainties, a neutrino oscillation experiment can be characterized by the total number of neutrino interactions observed (and hence the minimum observable $\left.P\left(\nu_{1} \rightarrow \nu_{2}\right)\right)$ and the average $\mathrm{L} / \mathrm{E}$ for the interacting neutrinos. These parameters are summarized in Table 1 for the four neutrino oscillation experimental configurations discussed in the previous section.

Consider first the very long baseline (Fermilab $\rightarrow$ Gran Sasso) experiment. The mean value of $\mathrm{L} / \mathrm{E}$ for the interacting $\nu_{e}$ is $744 \mathrm{~km} / \mathrm{GeV}$, and the minimum observable $P\left(\nu_{e} \rightarrow \nu_{\mu}\right)$ is $\mathrm{O}\left(10^{-3}\right)$. Examples of the predicted charged current $e^{-}$and $\mu^{-}$spectra are shown in Fig. 7 for $\sin ^{2}(2 \theta)=1$ and four choices of $\Delta m^{2}$ in the range $5 \times 10^{-4}<\Delta m^{2}<4 \times 10^{-3} \mathrm{eV}^{2} / \mathrm{c}^{4}$. The spectra are clearly sensitive to values of the oscillation parameters within this range.

Figure 8 compares the $\nu_{e}-\nu_{\mu}$ oscillation single-event sensitivity contour in the $\left(\Delta m^{2}, \sin ^{2}(2 \theta)\right)$-plane for the very long baseline configuration with the corresponding contours for the three other configurations discussed in the previous sections. The very long baseline single - event contour extends down to $\Delta m^{2} \sim 3 \times 10^{-5} \mathrm{eV}^{2} / \mathrm{c}^{4}$ for $\sin ^{2}(2 \theta)=1$, with the "knee" in the contour at $\sin ^{2}(2 \theta) \sim 10^{-3}$. The predicted single-event contours for the two long baseline 
(Fermilab $\rightarrow$ Soudan) configurations have similar $\Delta m^{2}$ reaches as $\sin ^{2}(2 \theta) \rightarrow 1$, and have "knees" at lower values of $\sin ^{2}(2 \theta)$ reflecting the larger charged current event statistics. Note that the $\Delta m^{2}$ reaches of the long- and very-longbaseline configurations are more than an order of magnitude better than the expected reaches of the next generation of currently proposed neutrino experiments [11]-[20]. The single event contour for the short baseline configuration exhibits a $\Delta m^{2}$ reach that is only a little better than the expected sensitivities of the next generation of long-baseline neutrino experiments. However, the very large charged current event rates expected for the short baseline configuration would enable sensitivities approaching $\sin ^{2}(2 \theta) \sim 10^{-7}$ for large $\Delta m^{2}$ $\left(\Delta m^{2} \sim 1 e V^{2} / c^{4}\right)$. This $\sin ^{2}(2 \theta)$ reach is almost a factor of 1000 better than the expected reaches of presently proposed future experiments, but would only be attained in the absence of backgrounds from, for example, secondary production of neutrinos from interactions in the vacinity of the experiment, or a component of neutrinos produced from the decays of "wrong-sign" muons produced in the accelerator complex upstream of the storage ring. Finally, Fig. 9 shows the single-event contours in the $\left(\Delta m^{2}, \sin ^{2}(2 \theta)\right)$-plane for $\nu_{e}-\nu_{\tau}$ oscillations, where once again the search is based on looking for wrong-sign muons produced in charged current interactions. The pure $\nu_{e}$ component in the neutrino beam from a muon storage ring would enable the sensitivity of $\nu_{e}-\nu_{\tau}$ searches to approach that of $\nu_{\mu}-\nu_{e}$ searches, and result in an improvement beyond the sensitivities of past $\nu_{e}-\nu_{\tau}$ oscillation searches [21, 22] by many orders of magnitude.

\section{Conclusions}

A very intense muon source of the type currently being developed for a future high-luminosity muon collider would provide sufficient muons to make very intense neutrino and antineutrino beams. If $\mathrm{O}\left(10^{20}\right)$ muons per year were allowed to decay within a $20 \mathrm{GeV} / \mathrm{c}$ storage ring with a straight section pointing in the desired direction, the resulting beams would produce hundreds of charged current neutrino interactions per year in a $10 \mathrm{kT}$ detector on the other side of the Earth. High beam intensities, together with the purity of the initial flavor content of the neutrinos and antineutrinos within the beam, would provide unique opportunities for short-, long-, and very-long-baseline neutrino experiments. The physics program that can be pursued with muon storage ring neutrino sources could begin with a short baseline experiment using relatively low energy muons $(\mathrm{O}(1 \mathrm{GeV}))$ as soon as an intense muon source became operational, and be extended to include higher energy muon storage rings and longer baseline experiments as higher energy muon beams became available.

\section{Acknowledgments}

I would like to thank Carol Johnstone, Ray Stefanski, and Alvin Tollestrup for valuable comments on the manuscript. This work was performed at the Fermi 
National Accelerator Laboratory, which is operated by Universities Research Association, under contract DE-AC02-76CH03000 with the U.S. Department of Energy. 


\section{References}

[1] " $\mu^{+} \mu^{-}$Collider Feasibility Study", The Muon Collider Collaboration, Fermilab-Conf-96/092, July 1996, unpublished.

[2] R. Palmer, A. Tollestrup, and A. Sessler, "Status Report of a High Luminosity Muon Collider and Future Research and Development Plans", Proc. of the 1996 DPF / DPB Summer Study on New Directions for High-energy Physics (Snowmass 96), Snowmass, CO, 25 June - 12 July 1996.

[3] A. De Rujula, S.L. Glashow, R.R. Wilson, and G. Charpak, Phys. Reports 99, 341 (1983).

[4] See for example: T. K. Gaisser, "Cosmic Rays and Particle Physics", Cambridge University Press 1990.

[5] C. Johnstone, private communication.

[6] C. Johnstone, "High Intensity Muon Storage Rings for Neutrino Production: Lattice Design", Proc. Workshop on Physics at the First Muon Collider and Front-End of a Muon Collider, Fermilab 6-9th November, 1997, Fermilab TM-2036, November 1997.

[7] S. Holmes et al., "A Development Plan for the Fermilab Proton Source", FERMILAB-TM-2021, September 1997, unpublished.

[8] A.N. Skrinsky and V.V. Parkhomchuk, Sov. J. Part. Nucl. 12, 223 (1981).

[9] R.M. Barnett et al. (Particle Data Group), Phys. Rev. D54, 261 (1996).

[10] See for example: F. Boehm and P. Vogel, "Physics of Massive Neutrinos", Cambridge University Press, 1987.

[11] E. Ables et al; "P-875: A Long-baseline Neutrino Oscillation Experiment at Fermilab, NuMI-L-63 Minos Proposal", Feb. 1995, unpublished.

[12] E. Church et al. (BooNE Collab.), "A letter of intent for an experiment to measure $\nu_{\mu} \rightarrow \nu_{e}$ oscillations and $\nu_{\mu}$ disappearance at the Fermilab Booster", May 16, 1997, unpublished.

[13] A.S. Ayan et al. (TOSCA Collab.), "A High Sensitivity Short Baseline Experiment to Search for $\nu_{\mu} \rightarrow \nu_{\tau}$ Oscillation", CERN-SPSC/97-5, March 1997, unpublished.

[14] K.Nishikawa et al. (KEK-PS E362 Collab.), "Proposal for a Long Baseline Neutrino Oscillation Experiment, using KEK-PS and Super-Kamiokande", 1995, unpublished; INS-924, April 1992, Submitted to J.Phys.Soc.Jap.

[15] M. Ambrosio et al. (NOE Collab.), Nucl. Instr. Meth. A363, 604 (1995).

[16] P. Ce Cennini et al., "ICARUS II: A Second Generation Proton Decay Experiment and Neutrino Observatory at the Gran Sasso Laboratoty: Proposal", LNGS-94/99-I (1994), unpublished.

[17] T. Ypsilantis, Nucl. Instr. Meth. A371, 330 (1996). 
[18] A. Rubbia et al. (ICARUS Collab.), CERN-SPSLC-96-58, Dec 1996, unpublished.

[19] G. Drexlin et al. (NESS Collab.), "Neutrinos at the European Spallation Source", unpublished.

[20] B. Bugg et al. (ORLANDO Collab.), "Prospect for a Neutrino Oscillation Experiment at the National Spallation Neutron Source", Proc. of the Intersections of Particle and Nuclear Physics Conference in Big Sky, MT in June 1997.

[21] N. Ushida et al. (The Fermilab E531 Collab.), Phys. Rev. Lett. 57, 2897 (1986).

[22] M. Talebzadeh et al. (BEBC WA66 Collab.), Nucl. Phys. B291, 503 (1987). 
Table 1: Summary of the neutrino oscillation experimental configurations considered in the text. The number of $\nu_{e}$ charged current interactions per year and the mean energies of the interacting neutrinos are listed for a detector of mass $m_{D E T}$ a distance $\mathrm{L}$ from a storage ring in which $7.5 \times 10^{20}$ unpolarized positive muons per year are injected with momenta $\mathrm{p}$, and $25 \%$ of the muons decay in the straight section pointing at the experiment.

\begin{tabular}{cccccc}
\hline \hline $\begin{array}{c}\mathrm{p} \\
(\mathrm{GeV} / \mathrm{c})\end{array}$ & $\begin{array}{c}m_{D E T} \\
(\mathrm{kT})\end{array}$ & $\begin{array}{c}\mathrm{L} \\
(\mathrm{km})\end{array}$ & $\begin{array}{c}\left\langle E_{\nu}>\right. \\
(\mathrm{GeV})\end{array}$ & $\begin{array}{c}\mathrm{L} /<E_{\nu}> \\
(\mathrm{km} / \mathrm{GeV})\end{array}$ & $\begin{array}{c}\nu_{e} \mathrm{CC} \\
\text { interactions } / \mathrm{yr}\end{array}$ \\
\hline 20 & 10 & 9900 & 13 & 744 & $1 \times 10^{3}$ \\
20 & 10 & 732 & 13 & 57 & $2 \times 10^{5}$ \\
10 & 10 & 732 & 6.6 & 111 & $3 \times 10^{4}$ \\
1.5 & 1 & 1 & 1 & 1 & $5 \times 10^{6}$ \\
\hline \hline
\end{tabular}




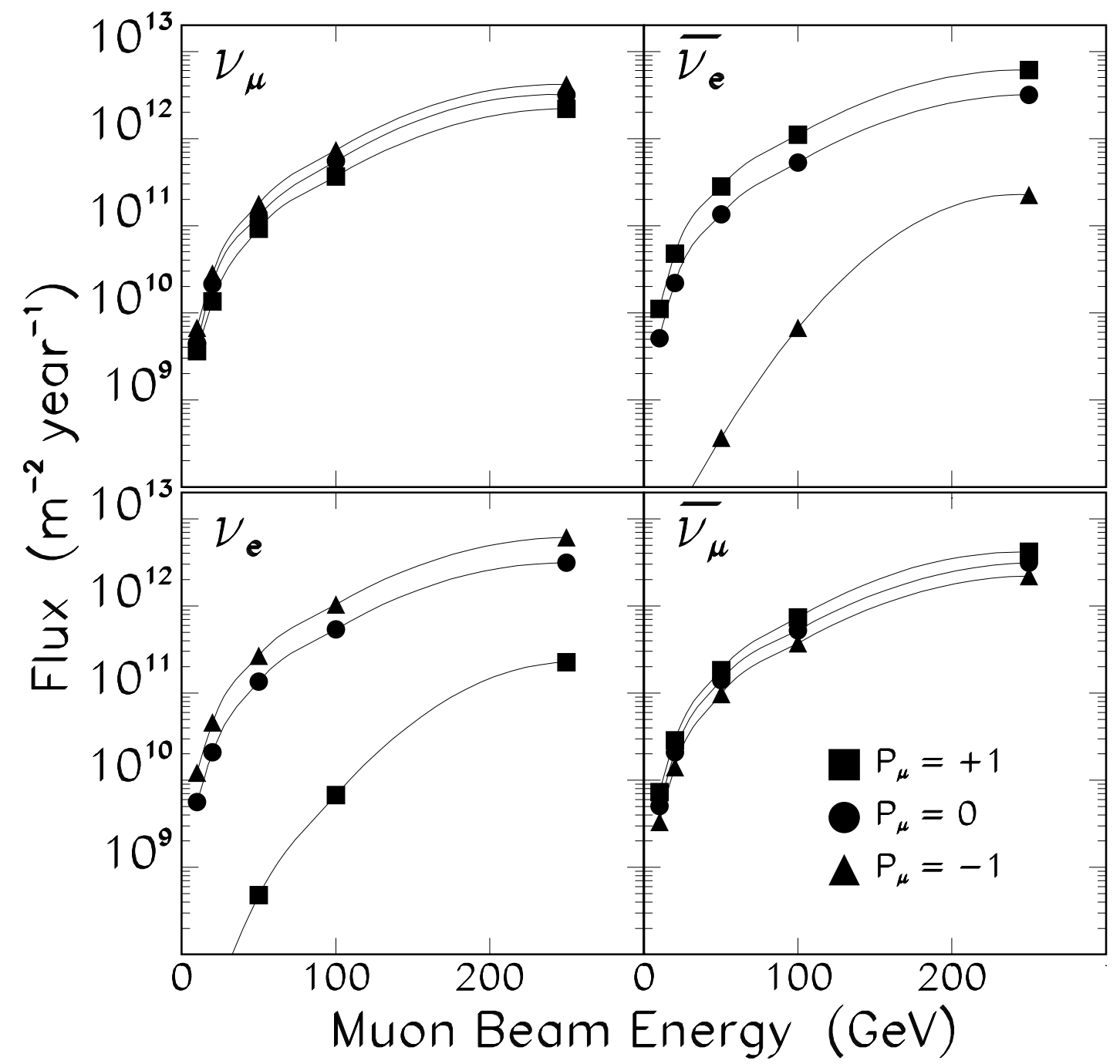

Figure 1: Calculated neutrino and antineutrino fluxes at a far site located $9900 \mathrm{~km}$ from a muon storage ring neutrino source (e.g. Fermilab $\rightarrow$ Gran Sasso). The parameters of the muon storage ring are described in the text. The fluxes are shown as a function of the energy of the stored muons for negative muons (top two plots) and positive muons (bottom two plots), and for three muon polarizations as indicated. 


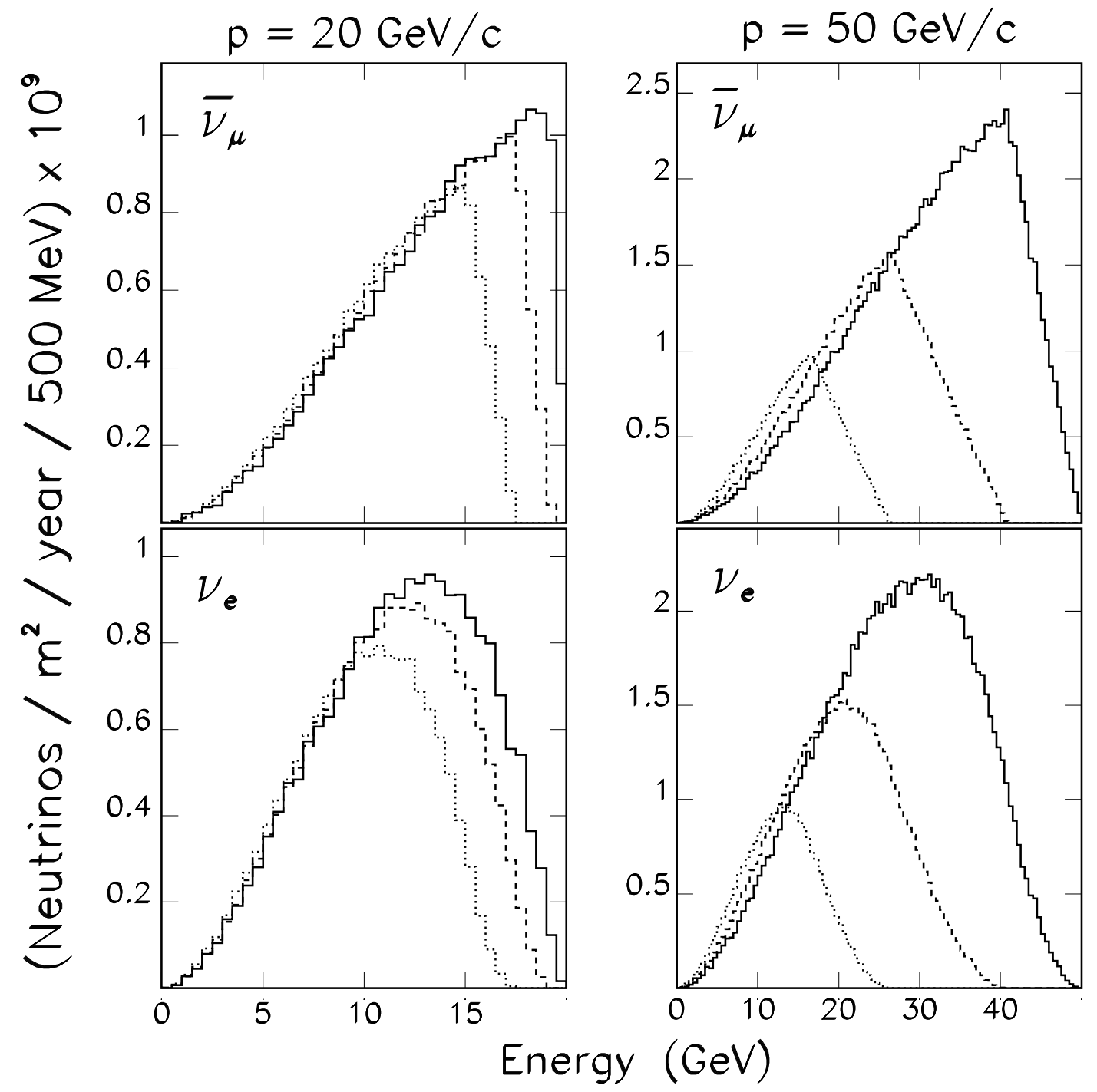

Figure 2: Calculated neutrino and antineutrino differential spectra at a far site located $9900 \mathrm{~km}$ from a muon storage ring neutrino source (e.g. Fermilab $\rightarrow$ Gran Sasso). The parameters of the muon storage ring are described in the text. The spectra correspond to unpolarized positive muons circulating in the muon storage ring with momenta of $20 \mathrm{GeV} / \mathrm{c}$ (left plots) and $50 \mathrm{GeV} / \mathrm{c}$ (right plots). The solid curves are obtained by averaging the fluxes over a central "spot" with opening angle $\Delta \theta=1 \mathrm{mr}$. The dashed and dotted curves are obtained by averaging over annuli centered on the beam axis and covering the angular intervals $1<\Delta \theta<2 \mathrm{mr}$ and $2<\Delta \theta<3 \mathrm{mr}$ respectively. 


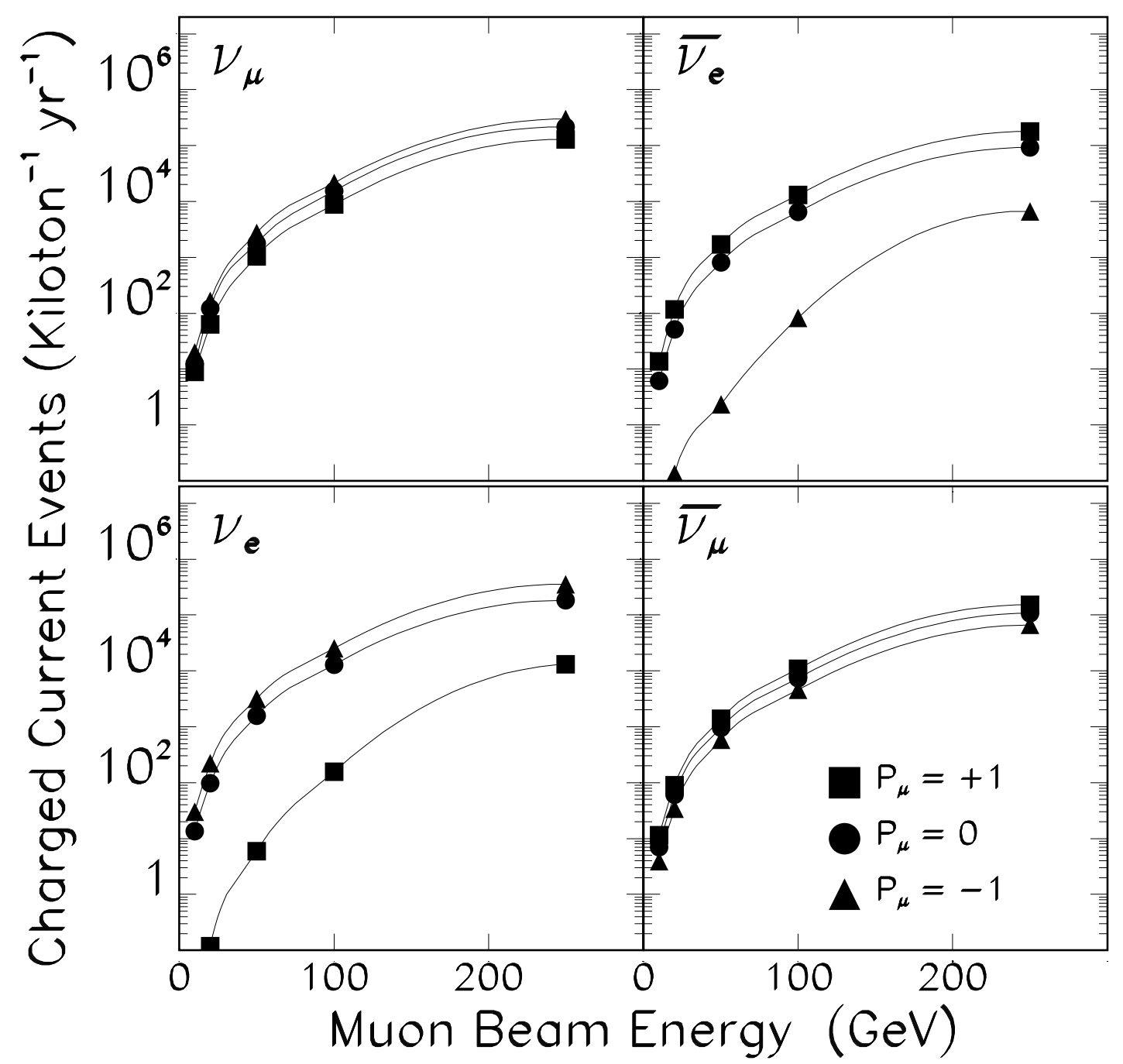

Figure 3: Calculated neutrino and antineutrino charged current interaction rates in a detector located $9900 \mathrm{~km}$ from a muon storage ring neutrino source (e.g. Fermilab $\rightarrow$ Gran Sasso). The parameters of the muon storage ring are described in the text. The rates are shown as a function of the energy of the stored muons for negative muons (top two plots) and positive muons (bottom two plots), and for three muon polarizations as indicated. 


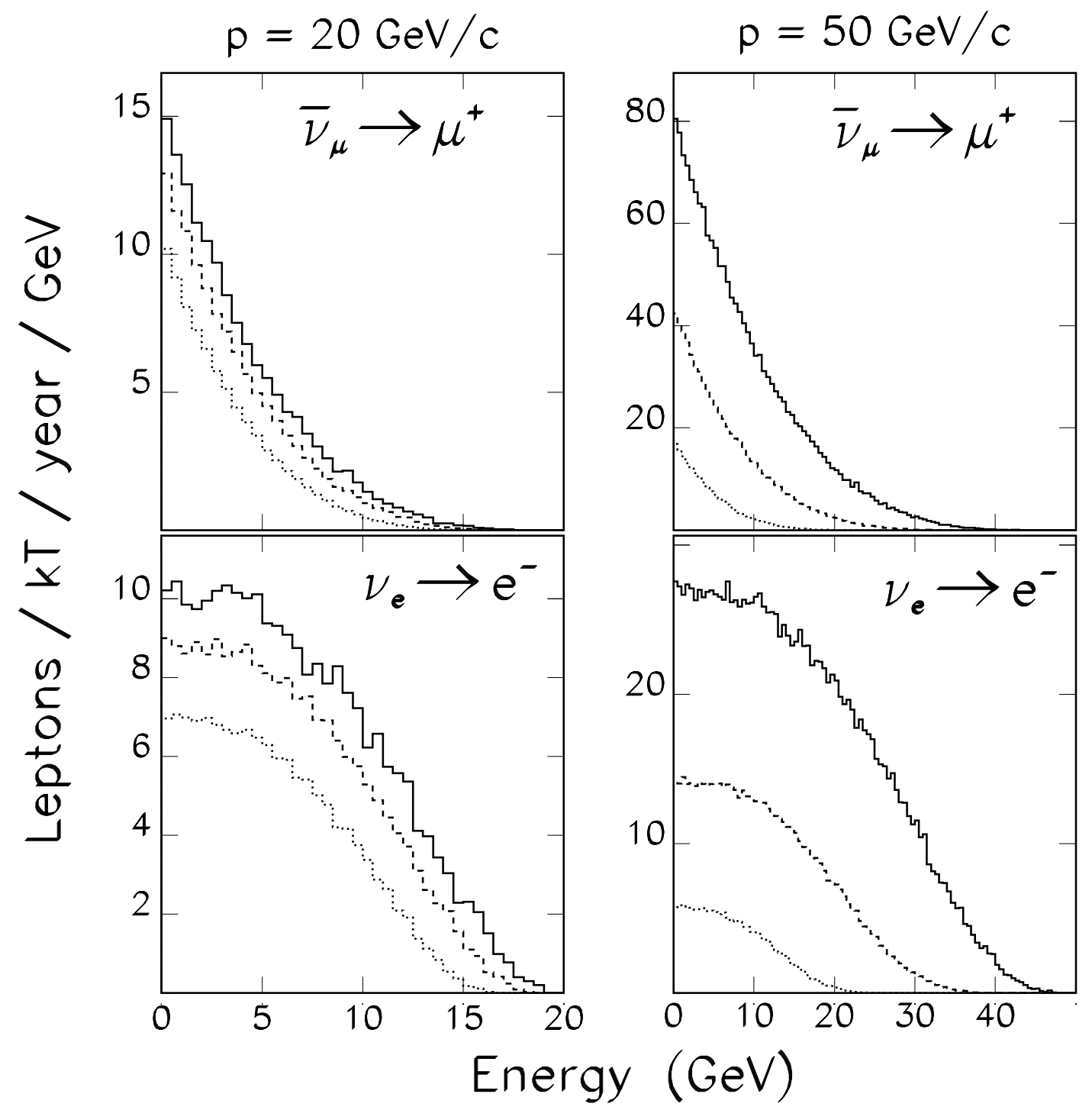

Figure 4: Calculated lepton and antilepton differential spectra for particles produced in charged current interactions in a detector located $9900 \mathrm{~km}$ from a muon storage ring neutrino source (e.g. Fermilab $\rightarrow$ Gran Sasso). The parameters of the muon storage ring are described in the text. The spectra correspond to unpolarized positive muons circulating in the muon storage ring with momenta of $20 \mathrm{GeV} / \mathrm{c}$ (left plots) and $50 \mathrm{GeV} / \mathrm{c}$ (right plots). The solid curves are obtained by averaging the fluxes over a central "spot" with opening angle $\Delta \theta=1 \mathrm{mr}$. The dashed and dotted curves are obtained by averaging over annuli centered on the beam axis and covering the angular intervals $1<\Delta \theta<2 \mathrm{mr}$ and $2<\Delta \theta<3 \mathrm{mr}$ respectively. 


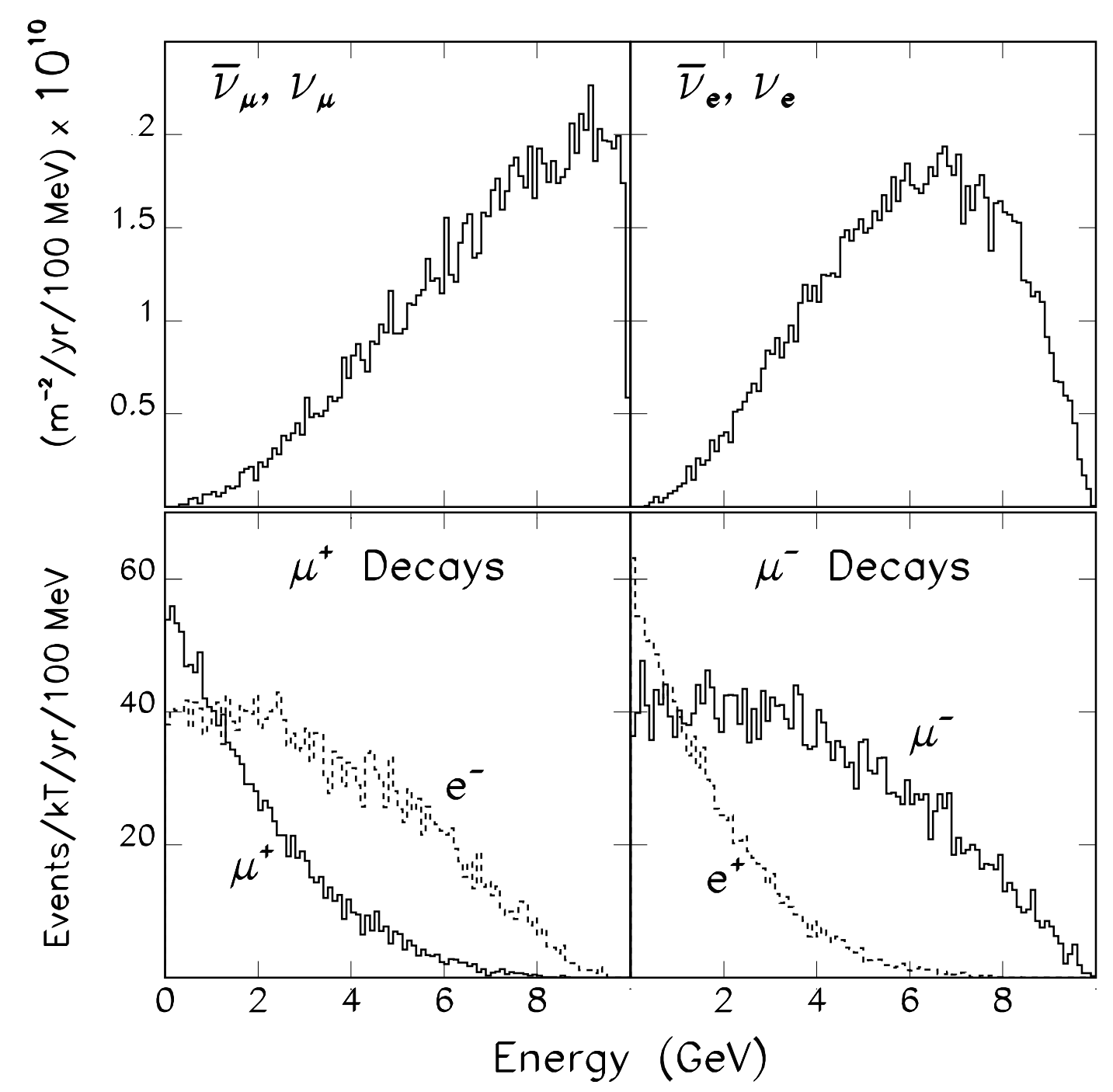

Figure 5: Calculated fluxes and spectra in a detector $732 \mathrm{~km}$ downstream of a muon storage ring neutrino source in which $10 \mathrm{GeV} / \mathrm{c}$ unpolarized muons are circulating. The top two plots show the neutrino and antineutrino spectra, and the bottom two plots show the charged lepton spectra from charged current interactions when positive muons (bottom left) and negative muons (bottom right) are stored in the ring. 


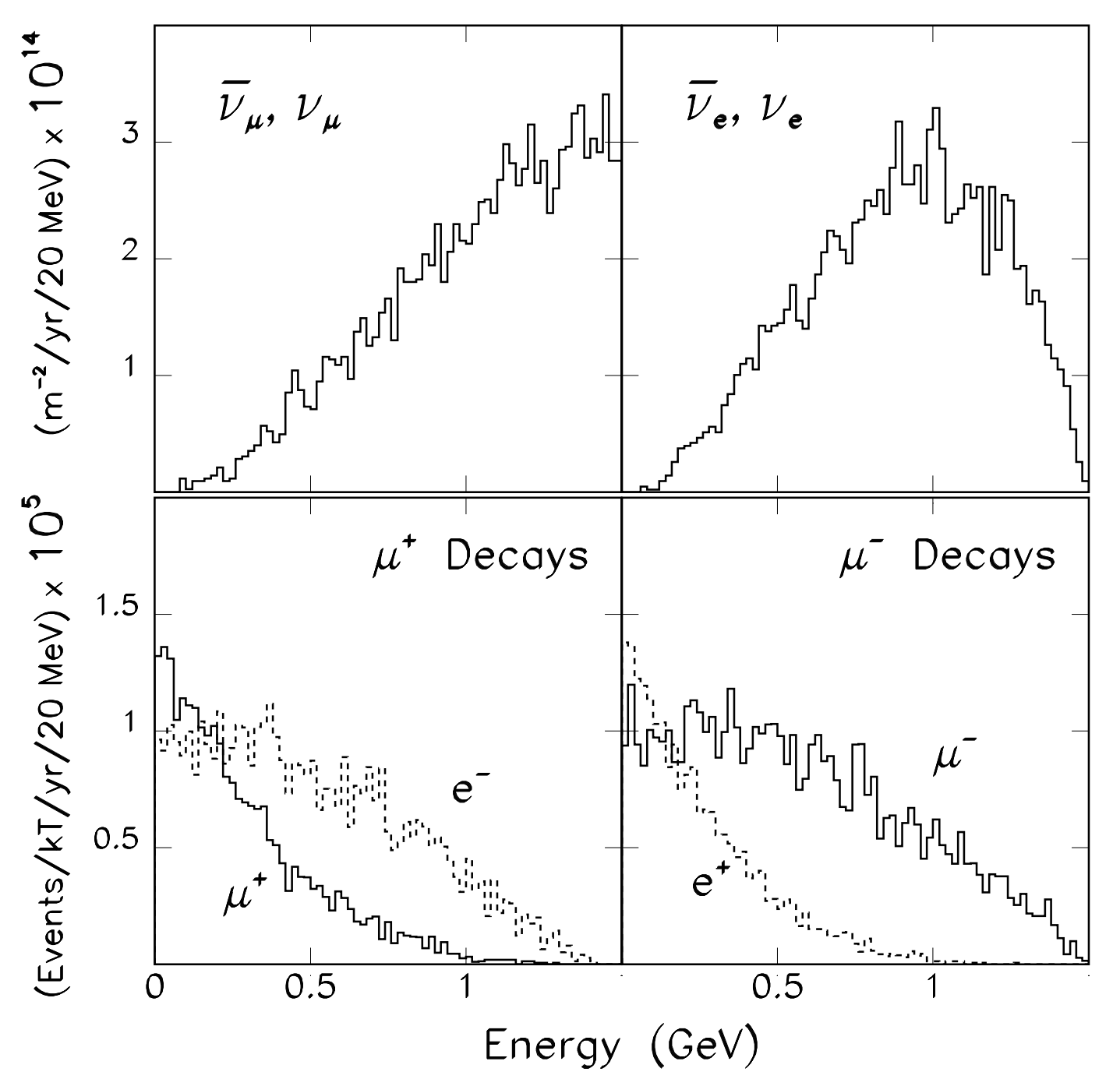

Figure 6: Calculated fluxes and spectra in a detector $1 \mathrm{~km}$ downstream of a muon storage ring neutrino source in which $1.5 \mathrm{GeV} / \mathrm{c}$ unpolarized muons are circulating. The top two plots show the neutrino and antineutrino spectra, and the bottom two plots show the charged lepton spectra from charged current interactions when positive muons (bottom left) and negative muons (bottom right) are stored in the ring. 


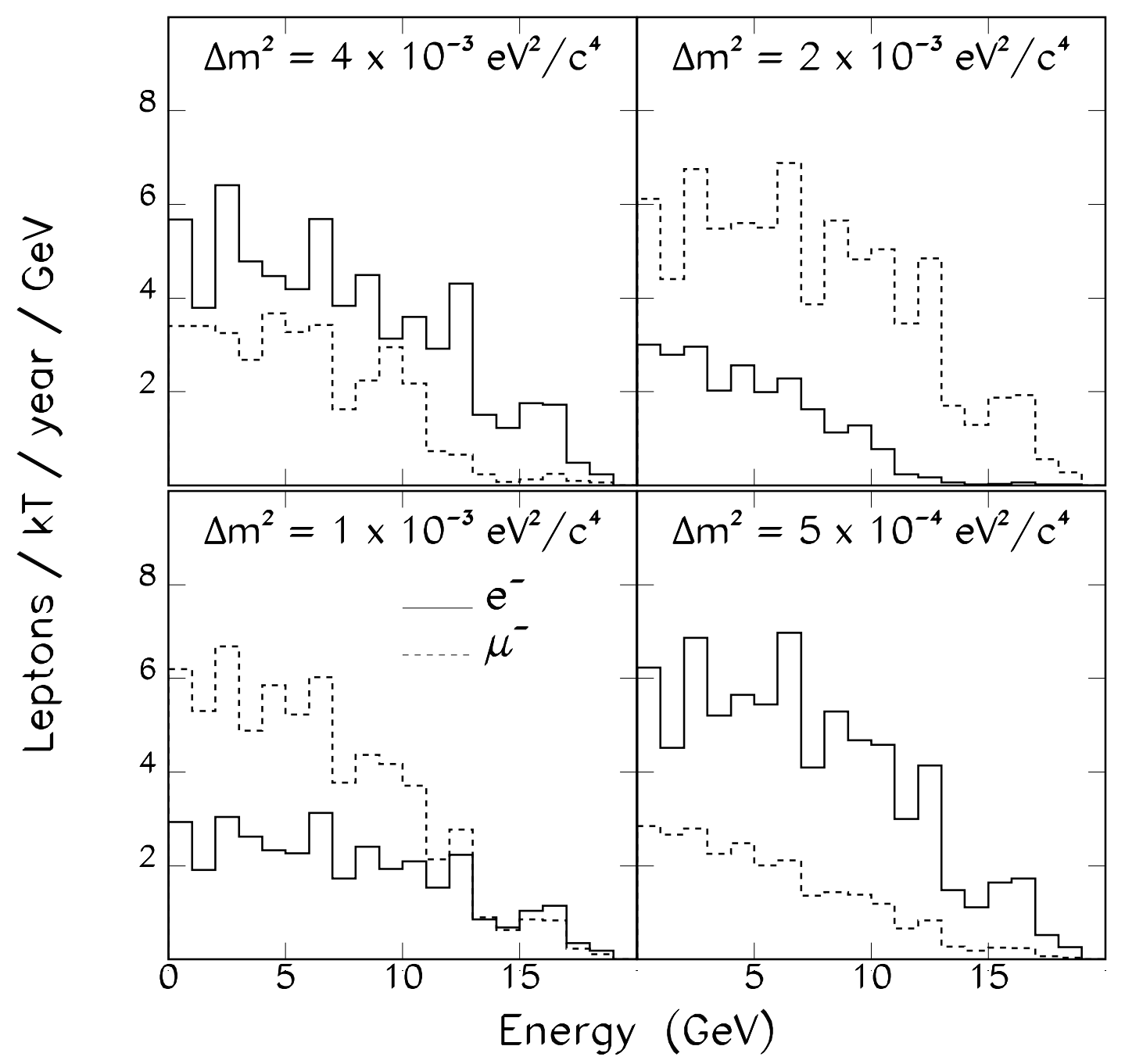

Figure 7: Predicted charged lepton energy distributions in a detector $9900 \mathrm{~km}$ from a ring in which $20 \mathrm{GeV}$ unpolarized positive muons are decaying. The predicted differential distributions for $\mathrm{e}^{-}$(solid histograms) and $\mu^{-}$(broken histograms) are shown assuming $\nu_{e}-\nu_{\mu}$ oscillations are occurring with $\sin ^{2} 2 \theta=1$ and $\Delta m^{2}$ as indicated on the 4 sub-plots. 


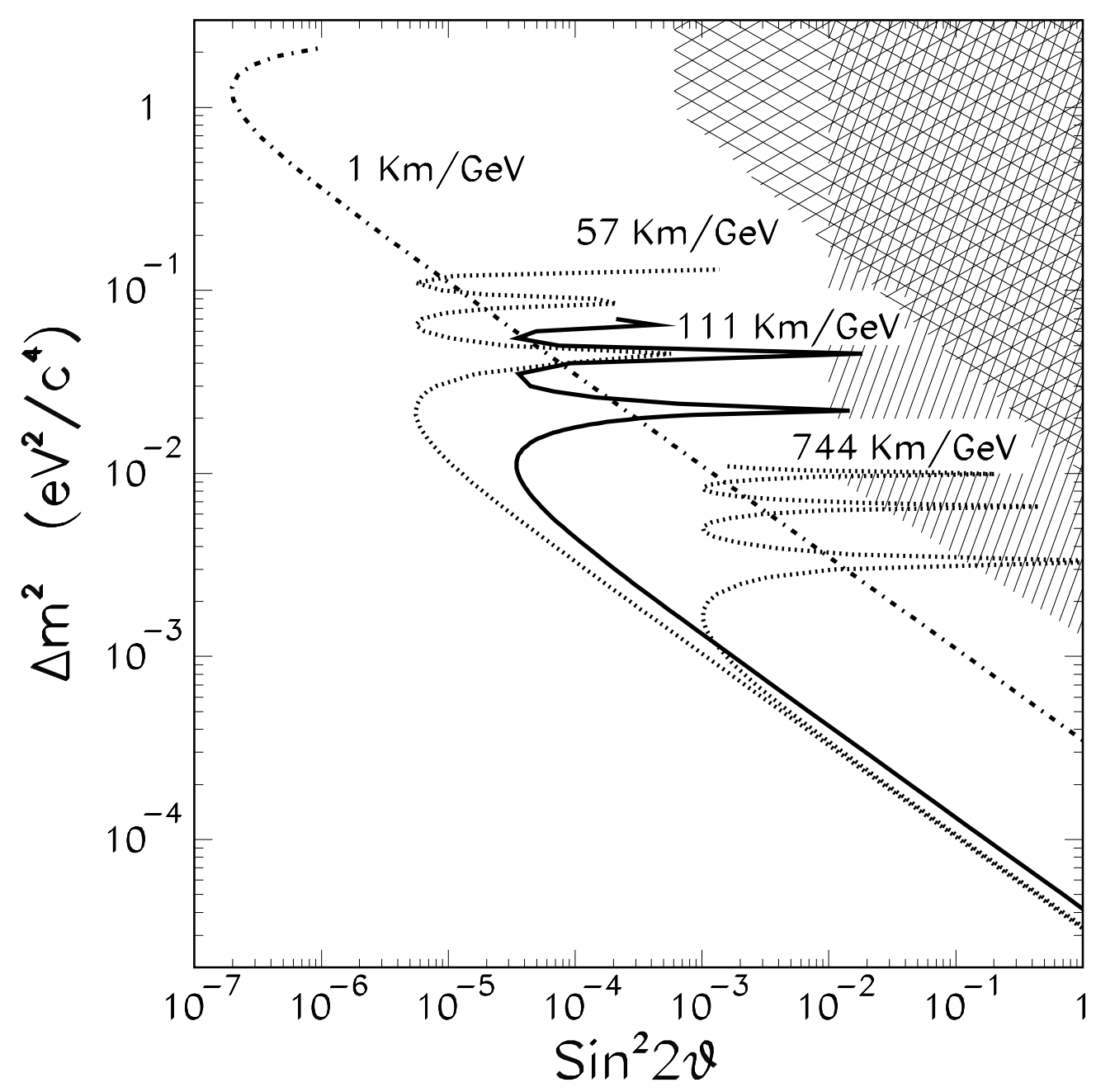

Figure 8: Contours of single-event sensitivity for $\nu_{e}-\nu_{\mu}$ oscillations for 1 year of running with the 4 values of $\mathrm{L} / \mathrm{E}$ specified on the figure, which correspond to the 4 detector configurations summarized in Table 1. The hatched and cross-hatched areas show the expected regions that will be explored by respectively the MINOS experiment [10] after 2 years of running and the MiniBooNe experiment [11] after 1 year of running. 


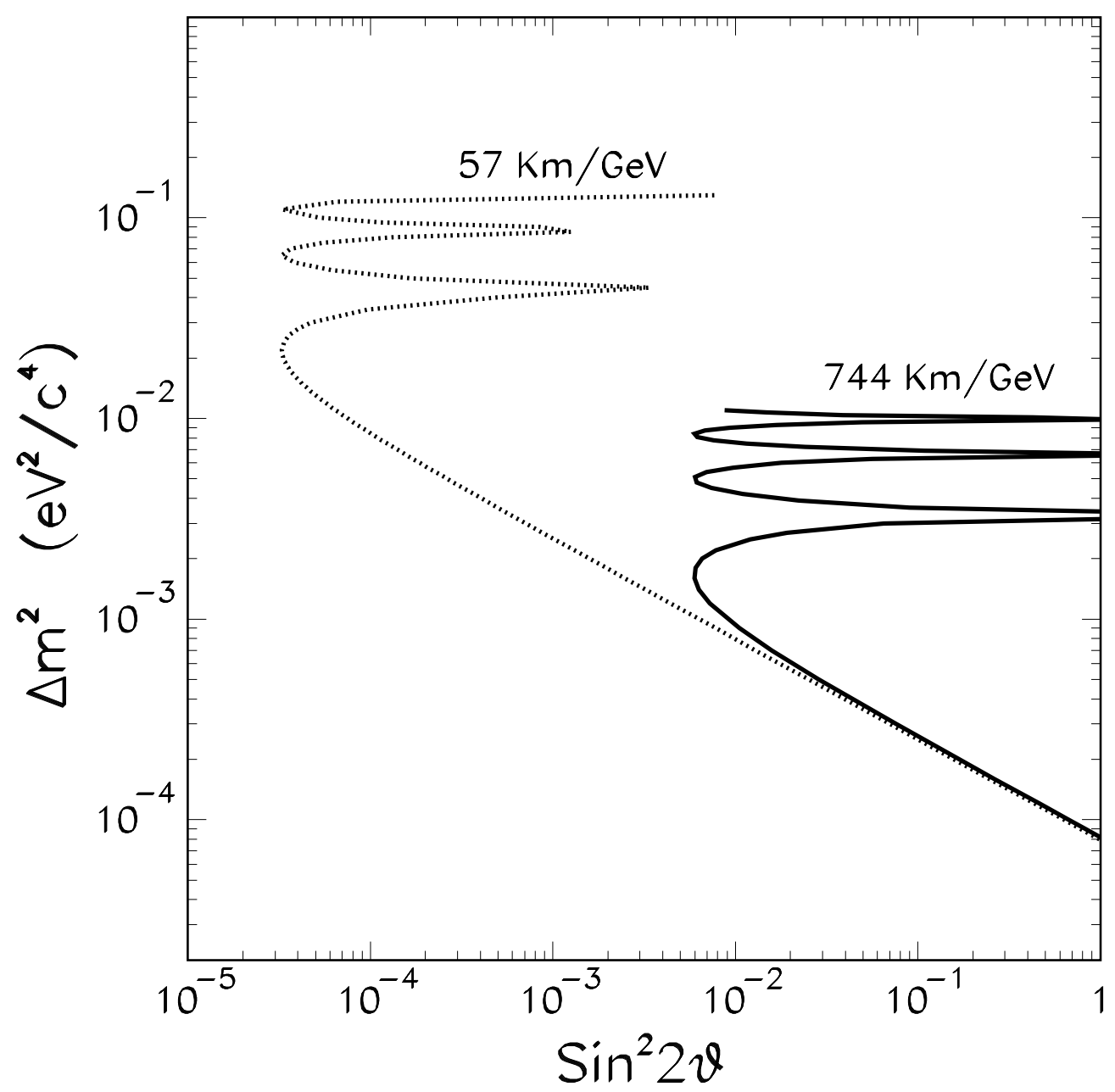

Figure 9: Contours of single-event sensitivity for $\nu_{e}-\nu_{\tau}$ oscillations based on searching for "wrong-sign" muons with the very-long-baseline (solid contour) and longbaseline (dotted contour) configurations listed in Table 1 for the average L/E values of respectively $744 \mathrm{~km} / \mathrm{GeV}$ and $57 \mathrm{~km} / \mathrm{GeV}$. The contours correspond to a $10 \mathrm{kT}$ year exposure with $20 \mathrm{GeV} / \mathrm{c}$ unpolarized muons stored in the ring and the straight section pointing at detectors $9900 \mathrm{~km}$ and $732 \mathrm{~km}$ from the ring. 